\title{
Treatment of physical disorder in children with mental disorder: A health care utilization study
}

\author{
Harleen K Ghuttora' ${ }^{1}$, David RL Cawthorpe ${ }^{2}$ \\ 1. O'Brien Center, Faculty of Medicine, University of Calgary, Canada. 2. Faculty of Medicine, Departments of Psychiatry \\ and Community Health Sciences, University of Calgary, Canada.
}

Correspondence: David RL Cawthorpe. PhD, Adjunct Associate Professor. Address: Richmond Road Diagnostic and Treatment Center, 1820 Richmond Road S.W. Calgary, Alberta, T2T 5C7, Canada. E-mail: cawthord@ucalgary.ca

Received: July 28, 2013

DOI : $10.5430 /$ jha.v3n2p24

Online Published: October 29, 2013

Accepted: October 16, 2013

URL: http://dx.doi.org/10.5430/jha.v3n2p24

\section{Abstract}

Objective: This study examines, across physician billing, ambulatory and inpatient/emergency datasets, the health care utilization of individuals under the age of 18 years for physical disorders in relationship to the existence of a physician assigned psychiatric disorder.

Methods: A retrospective sample of all visit records from three datasets (physician billing, ambulatory records, and inpatient/emergency records $n=12687710)$ was constructed for cases $(n=26392)$ and comparisons $(n=205281)$. The mean number of visits for physical disorders (excluding psychiatric disorders) was calculated for groups defined as cases and comparisons with and without psychiatric disorders.

Results: Among Cases and Comparisons with and without psychiatric disorders, physical disorders are significantly greater for any with psychiatric disorder over the 16 years study period in both physician billing and ambulatory datasets. This result differs in the inpatient/emergency dataset in that cases have about 1/3 the number of admissions for physical diagnoses.

Conclusion: It was unexpected that cases with a psychiatric diagnosis in the physician billing dataset had fewer physical disorder related inpatient and emergency admissions. We explore the putative explanations for the observed treatment bias related to physical disorders of children with psychiatric disorders.

\section{Key words}

Stigmatization, Utilization of services, Psychiatric diagnosis, Population study

\section{I ntroduction}

Stigma and discrimination are a national concern among children and adolescent with mental disorders. Canada spends $4.8 \%$ of the health budget on mental health services ${ }^{[1]}$ and yet only $20 \%$ of the children diagnosed with a psychiatric disorder (PD) receive mental health specialty services ${ }^{[2,3]}$. The insufficient usage of special mental health services can be credited to availability of resources and/or the caregiver's reluctance to seek treatment for the child due to societal stigma. Many studies have focused on discussing the caregiver's role in seeking treatment for children with psychiatric disorder and how it is affected by societal stigma ${ }^{[2,4,5]}$. For example, Pescosolido et al. ${ }^{[2]}$ reported that three out of four youth experiencing depression are not diagnosed and of those diagnosed only 30\% receive treatment. Further findings suggest 
approximately $40 \%-60 \%$ patients in treatment terminate it prematurely ${ }^{[5,6]}$. While the reasons for such findings remain under debate, Heflinger and Hinshaw ${ }^{[7]}$ present one explanation based on professional and institutional stigma that exists for child and adolescent patients seeking help for mental disorders. Evidently, system-based stigma and societal stigma are a great barrier to the treatment of psychiatric disorders in children.

In spite of access issues, the rate of mental disorders has doubled between 1994 and 2009 from $4.7 \%$ to $10 \%{ }^{[8]}$. This doubling could be due to a true increase in population, pharmacological marketing and inventions, and/or better education for physicians as well as families. Wilkes et al. ${ }^{[9]}$ have recently investigated the health costs for the treatment of physical disorders in PD diagnosed children. The cost of treating physical disorders for children with PD was found to be 2X higher than those without a PD ${ }^{[9]}$. With increasing mental disorder diagnoses and health care costs, it is essential to examine this effect on children diagnosed with a PD experiencing physical disorders. While Newton et al. ${ }^{[10]}$ have examined the mental diagnoses in emergency departments in Alberta, the present study extends this conceptualization to include overall health care utilization patterns by children and youth under the age of 18. In this study we examine the health care service utilization for physical disorders of children with psychiatric diagnosis across three health care settings; physician billing, ambulatory services, and inpatient/emergency services. As the cost of treating physical disorder for children with PD is double that of children without a PD, the children with PD were expected to have the highest number of visits in all three health care settings, which was not the case.

\section{Methods}

A retrospective case-comparison design was used to evaluate health care utilization in the area of Calgary, Alberta for individuals under the age of 18 over a 16-year study period from 1994 to 2009 fiscal years. The values reported represent counts or averages of cumulative prevalence over the measurement period. Younger children have a longer period of access compared to older children, hence the average number of visits by age group diminishes as age increases. However, comparisons are made within age groupings across settings thereby truncating the effects of shorter period of access for older children.

Datasets from three health care settings; physician billing, ambulatory records, and inpatient/emergency records were compiled for both the case and comparison groups matched on age and sex with a ratio of 1:8 for cases to comparisons. Based on these datasets, we compared the average number of visits per unique individual for non-psychiatric physical diagnoses for both the case and comparison groups. The physical disorders included any physician assigned ICD physical (non-psychiatric) disorder. The case and comparison groups differed on basis of registration in specialized mental health services in ambulatory and inpatient/emergency health care settings. These specialized mental health services include ambulatory clinics for children and families, inpatient child and adolescent treatment units, and emergency mental health services at both child and adult facilities.

The case group comprised of health care users who had at least once accessed the specialized mental health system and were registered in publicly funded mental health services between the years 1994-2009. The comparison group was matched with cases (8:1) based on age and sex and comprised of randomly selected health care users who had never been registered in publicly funded mental health services between the years 1994-2009 and had not received specialized mental health care. These groups were further divided by the presence of a physician-assigned psychiatric diagnosis. Therefore, four study groups emerged for each dataset; Case PD, Case non-PD, Comparison PD, and Comparison non-PD. The PD group included cases or comparisons with any physician-assigned ICD psychiatric disorder. The non-PD group included cases or comparisons without a physician assigned ICD psychiatric disorder.

The four study groups defined the dependent variable whereas the counts of physical disorders constituted the main independent variable and age was the main demographic variable considered in the analysis. These variables were compared across the three health care settings. The mean numbers of physical disorder related visits for the dependent variable groupings by age and by health care setting were the main data representations. 
The Case PD group included individuals with a physician assigned psychiatric disorder who had received specialized mental health services in ambulatory and inpatient/emergency settings. The Case non-PD group comprised of individuals without a physician assigned psychiatric diagnosis who nevertheless had accessed the specialized mental health care system. This individual could be a family member of a Case PD attending family therapy who was admitted to ambulatory/inpatient/emergency mental health clinic without assignment of a psychiatric disorder (false positive). The Comparison PD group included individuals with a physician assigned psychiatric diagnosis who had not received specialized mental health services. The Comparison non-PD group comprised of individuals without a physician assigned psychiatric diagnosis who had also not received specialized mental health services.

Three datasets were constructed with physician billing as the largest dataset as most individuals visit doctor's offices in community settings. The next largest data set is specialized ambulatory care and inpatient/emergency constituted the smallest data set. The ambulatory dataset represents access to specialized publicly funded hospitals and community clinics. Emergency and inpatient data represent admissions to hospital-based inpatient and emergency services. Visits by unique individuals to each of these services represent the basis of comparison across these datasets. Notwithstanding, inpatient visits were associated with greater contiguous length of stay and cost.

The physician billing dataset comprised of 10,693,374 billing records for a total of 231,673 unique individuals under the age of 18 from April 1, 1994 to March 31, 2010. This included 18,858 Case PD (8.1\%), 7,534 Case non-PD (3.3\%), 150,380 Comparison non-PD (64.9\%), and 54,901 Comparison PD (23.7\%). Table 1 displays further information regarding billing records and the number of individuals in the physician billing dataset.

Table 1. Physician billing dataset for the four study groups

\begin{tabular}{llllll}
\hline & & $\begin{array}{l}\text { Number of unique } \\
\text { individuals }\end{array}$ & $\begin{array}{l}\text { Percent of unique } \\
\text { individuals (\%) }\end{array}$ & $\begin{array}{l}\text { Number of Billing } \\
\text { records }\end{array}$ & $\begin{array}{l}\text { Percentage of Billing } \\
\text { records (\%) }\end{array}$ \\
\hline \multirow{2}{*}{ Case } & PD* & 18,858 & 8.1 & $1,968,660$ & 18.4 \\
& non-PD* & 7,534 & 3.3 & & 81.6 \\
\multirow{2}{*}{ Comparison } & PD* $^{*}$ Total & 54,901 & 23.7 & $8,724,714$ & 100 \\
\hline
\end{tabular}

*PD - Psychiatric Disorder

The ambulatory dataset comprised of 1,898,133 service records for a total of 131,212 unique individuals under the age of 18 from July 1, 1996 to September 30, 2010. This included 10,692 Case PD (8.1\%), 9021 Case non-PD (6.9\%), 97,514 Comparison non-PD (74.3\%), and 13985 Comparison PD (10.7\%). Table 2 displays further information regarding service records and the number of individuals in the ambulatory dataset.

Table 2. Ambulatory dataset for the four study groups

\begin{tabular}{llllll}
\hline & & $\begin{array}{l}\text { Number of } \\
\text { individuals }\end{array}$ & $\begin{array}{l}\text { Pnique } \\
\text { individuals (\%) }\end{array}$ & $\begin{array}{l}\text { Number of } \\
\text { Service records }\end{array}$ & $\begin{array}{l}\text { Percentage of } \\
\text { Service records (\%) }\end{array}$ \\
\hline \multirow{2}{*}{ Case } & PD* & 10,692 & 8.1 & 612,097 & 32.2 \\
& non-PD* & 9,021 & 6.9 & & \\
\multirow{2}{*}{ Comparison } & PD* $^{*}$ & 13,985 & 10.7 & $1,286,133$ & 67.8 \\
& Non-PD* & 97,514 & 74.3 & & 100 \\
\hline
\end{tabular}

*PD - Psychiatric Disorder

The inpatient/emergency dataset comprised of 96,203 service records for a total of 65,162 unique individuals under the age of 18 from April 3, 1994 to October 27, 2010. This included 3,691 Case PD (5.7\%), 7,650 Case non-PD (11.7\%), 
51,941 Comparison non-PD (79.7\%), and 1,880 Comparison PD (2.9\%). Table 3 displays further information regarding service records and the number of individuals in the inpatient/emergency dataset.

Table 3. Inpatient/Emergency dataset for the four study groups

\begin{tabular}{llllll}
\hline & & $\begin{array}{l}\text { Number of } \\
\text { individuals }\end{array}$ & $\begin{array}{l}\text { Percent of unique } \\
\text { individuals (\%) }\end{array}$ & $\begin{array}{l}\text { Number of } \\
\text { Service records }\end{array}$ & $\begin{array}{l}\text { Percentage of Service } \\
\text { records (\%) }\end{array}$ \\
\hline \multirow{2}{*}{ Case } & PD* $^{*}$ & 3,691 & 5.7 & 19,279 & 20 \\
& non-PD* & 7,650 & 11.7 & & \\
Comparison & PD* $^{*}$ & 1,880 & 2.9 & 76,924 & 80 \\
Total & Non-PD* & 51,941 & 79.7 & & 100 \\
\hline
\end{tabular}

*PD - Psychiatric Disorder

Case and Comparison data was represented in terms of the overall 16 year average visit per unique individual across the three datasets. Age and sex were examined within groups across datasets for potential confounding effects on the average visits per unique individual. As the largest dataset, physician billing formed the basis of comparison among the datasets. All datasets were truncated at the age of 18 years in order to examine pediatric services only.

\section{Results}

The physician billing, ambulatory, and inpatient/emergency datasets were examined for physical disorder related visits by all four study groups. The physician billing dataset for physical disorder related visits included 231,673 individuals with a total of 7,956,687 visits. The final groups comprised of 18,858 Case PD with 964,641 visits, 7,534 Case non-PD with 192,646 visits, 54,901 Comparison PD with 2,578,291 visits, and 150,380 Comparison non-PD with 4,221,109 visits. The relative mean number of visits for each of the four study groups can be found in Table 4 .

The ambulatory dataset for physical health related visits included 131,212 individuals with a total of 1,506,240 visits. This comprised of 10,563 Case PD with 289,752 visits, 9,150 Case non-PD with 79,142 visits, 13,449 Comparison PD with 417,612 visits, and 98,050 Comparison non-PD with 719,734 visits. The relative mean number of visits for each of the four study groups can be found in Table 4.

The inpatient/emergency dataset for physical health related visits included 65,162 individuals with a total of 88,010 visits. This comprised of 3,608 Case PD with 2,128 visits, 7,733 Case non-PD with 11,412 visits, 1,660 Comparison PD with 2,564 visits, and 52,161 Comparison non-PD with 71,906 visits. The relative mean number of visits for each of the four study groups can be found in Table 4.

The overall mean visits per unique individual were highest in the physician billing dataset, followed by ambulatory services dataset, and lowest in the inpatient/emergency services dataset. Similarly, the mean visits per unique individual in the Case PD group changed dramatically across the three health care settings. The Case PD group had the highest mean visit in physician billing (51.2), and unexpectedly lower in ambulatory services (27.4), and lowest in inpatient/emergency services (0.6) compared to the other four study groups.

Figures 1-3 illustrate the relationship between the four study groups in terms of age across the three health care settings. The figures report the differences in mean visits/admissions for physical disorders across the three health care settings between Cases and Comparisons comparing within age groups given the presence or absence of physician assigned PD. Comparisons were made within age group between the three health care settings. The graph lines depict each age group represented in the three figures. Lines provide the most direct means of illustrating comparisons within age groups across 
the three settings. Because each age group is identically defined across cases and comparisons with and without mental disorder, comparisons of the average number of visits within age grouping between figures are unbiased.

Table 4. Physical health-related visits to physician, ambulatory, and inpatient/emergency services

\begin{tabular}{lllll}
\hline Health Care Service & Study group & Individuals & Mean Visits & Total Visits \\
\hline \multirow{4}{*}{ Physician Billing } & Case PD* & 18,858 & 51.15 & 964,641 \\
& Case non-PD* & 7,534 & 44.35 & 192,646 \\
& Comparison PD* & 54,901 & 46.96 & $2,578,291$ \\
& Comparison non-PD* & 150,380 & 28.07 & $4,221,109$ \\
& Total & 231,673 & 34.344 & $7,956,687$ \\
& Case PD* & 10,563 & 27.4 & 79,142 \\
Ambulatory & Case non-PD* & 9150 & 8.6 & 417,612 \\
& Comparison PD* & 13,449 & 31.1 & 719,734 \\
& Comparison non-PD* & 98,050 & 7.3 & $1,506,240$ \\
& Total & 131,212 & 11.5 & 2,128 \\
Inpatient/Emergency & Case PD* & 3,608 & 0.59 & 11,412 \\
& Case non-PD* & 7,733 & 1.476 & 2,564 \\
& Comparison PD* & 1,660 & 1.545 & 71,906 \\
& Comparison non-PD* & 52,161 & 1.379 & 88,010 \\
\hline
\end{tabular}

*PD - Psychiatric Disorder
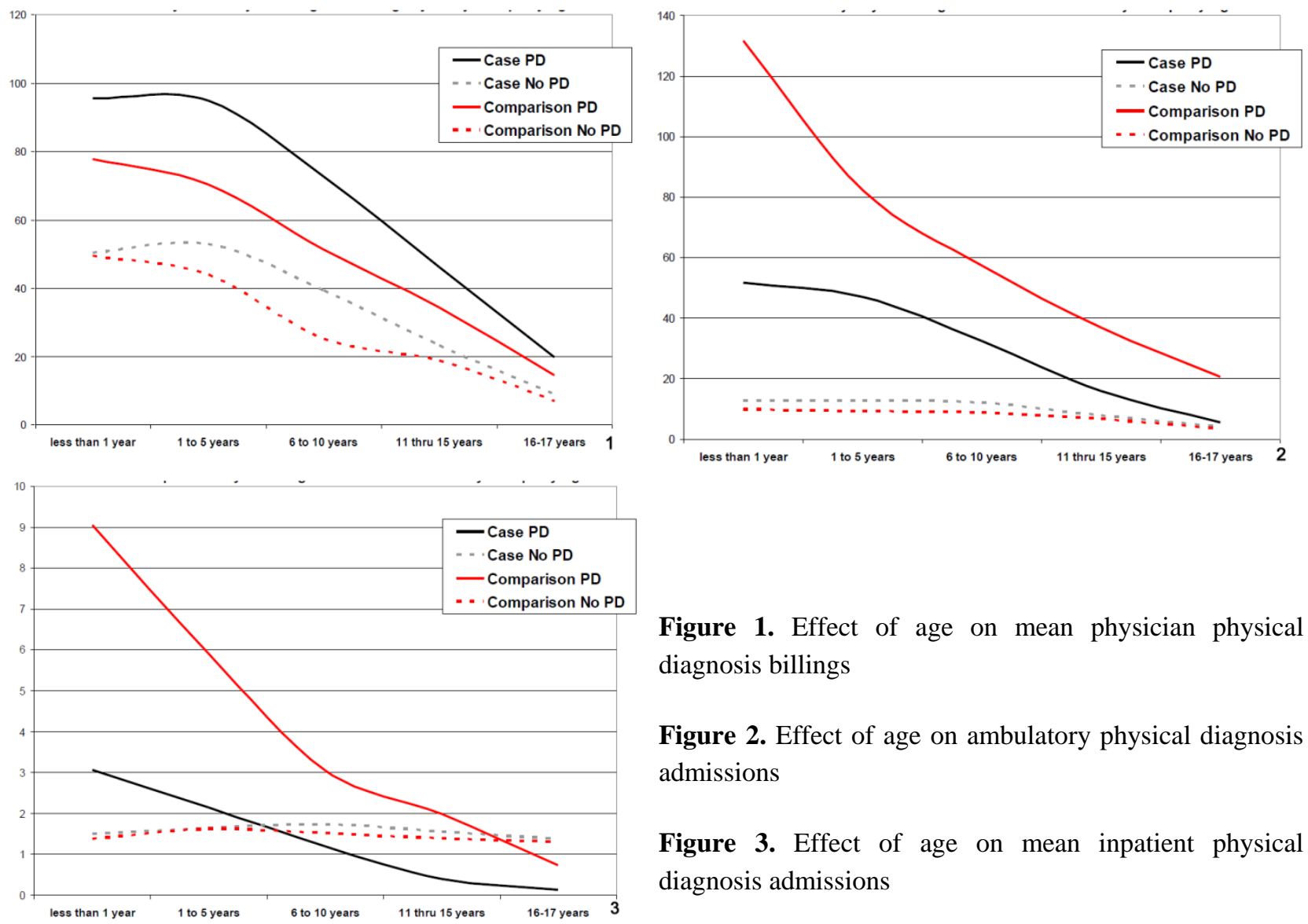

Figure 1. Effect of age on mean physician physical diagnosis billings

Figure 2. Effect of age on ambulatory physical diagnosis admissions

Figure 3. Effect of age on mean inpatient physical diagnosis admissions 
In each figure before the age of six all individuals with PD have higher proportions of physical disorders. In the ambulatory and inpatient/emergency dataset the Case PD group has less physical treatment visits compared to the physician billing dataset. The number of physical disorder related visits for groups with a physician assigned psychiatric disorder (Case PD and Comparison PD) drop below the groups without a physician assigned psychiatric disorder (Case non-PD and Comparison non-PD) in the inpatient/emergency setting. Individuals at the age of six in inpatient/emergency services show an inversion for the Case PD group and at the age of 16 years for the Comparison PD group showing less inpatient/emergency admissions than those without a PD (see Figure 3).

Age effects were observed within age groups comparing the dependent variable across the ambulatory and inpatient/ emergency and the physician billing datasets. Age effects were observed in the ambulatory dataset wherein Comparison PD and Case PD reversed at each age group (see Figure 2). Both were found to be greater than the two non-PD groups within each age grouping (see Figure 2). Age effects were observed in relationship to the average inpatient/emergency visits. For example, at the age of six, the average for Case PD dropped below all of the other study groups when represented as a function of age (< one year old, 0-five years old, six years-10 years old, 11 years-14 years old, and 15 years-17 years old) (see Figure 3).

The physician billing and ambulatory datasets show a reversed pattern for physical disorder related visits by the Case PD and Comparison PD study groups. In the physician billing dataset, Case PD have the highest mean visits (51.2) and the Comparison non-PD have the lowest mean visits (28.1). In the ambulatory billing dataset, the highest mean visit was by the Comparison PD group (31.1) and the lowest mean visit was by the Comparison non-PD group (7.3). The Case PD (27.4) had fewer visits than the Comparison PD (31.1) in the ambulatory services whereas it was the highest in the physician services. This represents a reversal from the physician billing dataset in health care utilization patterns between the two health care settings for the Case and Comparison PD study group. Overall, patients with a PD have higher mean visits for physical disorders when compared to individuals without a physician assigned PD in physician and ambulatory settings. However, the inpatient/emergency dataset differs from physician billing and ambulatory datasets and presents a paradox as it contains the highest mean visit by Comparison PD group (1.5) and the lowest mean visit by the Case PD group (0.6). The health care utilization in inpatient/emergency health care setting for the Case PD group was contrary to the health care utilization by the same group in the physician and ambulatory health care settings. These results indicate that patients from Case PD group diagnosed with a PD have the highest average visits to physician services for physical disorders but have the lowest average inpatient/emergency admissions among the four study groups.

\section{Discussion}

Through examination of the three health care setting datasets, the Case PD group was found to have the highest mean visits (51.2) to physician services for physical disorders but had lower mean visits (27.4) for the ambulatory services in relation to the Comparison PD group and the lowest mean visits (0.6) to inpatient/emergency services in comparison to all other study groups. The results of the ambulatory and inpatient/emergency settings contradict with the previously reported finding of children with psychiatric disorders having significantly more physical disorders ${ }^{[9]}$. Although, age and sex do not appear to confound or influence the overall findings, the effect of age primarily reveals that the reduction in access to health services for treatment of physical disorders is coincidental with the age of beginning school when most children are first diagnosed with psychiatric problems.

The changing health care utilization patterns by the Case PD group in comparison to the other study groups have several putative explanations. First, as indicated by the data, children may have fewer physical disorders at the level of ambulatory and inpatient/emergency services in comparison to the other study groups. This explanation is untenable as the Case PD individuals are seen having the highest physical disorders in the physician services. It is unlikely that the physical disorders decreased threefold in the inpatient/emergency dataset for the same individuals in comparison to the ambulatory and physician billing dataset results. 
Second, it is possible that the Case PD individuals may be receiving treatment from other private health care services. As private ambulatory and inpatient clinics are not readily available in the Calgary community, this is not plausible. Further, majority of the individuals studied are already using publicly funded system to obtain services for their PD at the level of physician billing and were diagnosed within the specialized mental health services.

Third, the individuals may be receiving treatment for their physical disorders from psychiatric clinics. Psychiatric clinics, however, are not usually equipped to treat these physical disorders and nor are psychiatrists usually qualified to treat serious physical disorders, hence excluding this possibility.

Fourth, it is possible that families of identified patients may have failed to respond to the physical needs of their children. But, the families have been supportive to the patient in terms of accessing physician services at a higher rate. Also, the large proportion of families having a child with mental disorder did get access to physician treatment for both psychiatric and physical disorders as well as ambulatory, inpatient, and emergency services ${ }^{[9]}$.

Fifth, the most parsimonious explanation of the observed decrease in treatment of the Case PD group is that this phenomenon represents a form of systematic bias in access to treatment for physical disorders that is directly related to the presence of a priori psychiatric disorder. The evidence in support of this interpretation within the inpatient/emergency dataset is the observation that access for physical problems is higher below the age of six and lower in all other age groups including both the non-PD groups and the Comparison PD group. This effect is not as obvious in the ambulatory dataset but nevertheless is present at every age in relation to the Comparison PD group and also represents a reversal from the observation that the Case PD group has the highest physical disorders in the physician billing dataset. Children tend to be diagnosed with psychiatric disorders less before the age of beginning school and more after as is indicated in the data.

In consideration of the published literature on system-based stigma ${ }^{[5,6]}$, the observed system bias (see Figure 3 : the precipitous drop of Case PD below all other groups at age six) as a form of stigma is supported by labeling theory ${ }^{[4]}$. The only difference between the Case PD group and Comparison PD group is that the Case PD individuals are identified as mental patients in regional ambulatory and inpatient information system as well as in their chart history on presentation to emergency or inpatient services. Additionally, patients and their families may self identify as mental patients on presentation to emergency/inpatient departments. Again, this interpretation is further supported by the observation that this bias does not exist before the age of six when psychiatric diagnosis is less likely.

The diagnosis and severity of physical disorder may be a potential confounder giving rise to differences in visits among the case and comparison groups. As a future perspective, further analysis is required on the type and severity of physical diagnosis for the case and comparison individuals giving insight into the presented systematic bias. A current limitation to this study is that no causal interpretations can be made from the results, for which a time-based analysis is required as the next step. Also, as a future implication, it may be of interest to examine the specific psychiatric and physical disorders that comprise the visits to the three health care settings. As this was beyond the focus of this paper, it may be examined in future studies.

\section{Conclusion}

Kutcher and Davidson ${ }^{[11]}$ and Wilkes and Cawthorpe ${ }^{[12]}$ state a need for more mental health resources at all levels of health care settings for treatment of mental health to be equitable. Further, Newton et al. ${ }^{[10]}$ have reported on the effect of sociodemographic factors on the number of visits to emergency services by mental health patients, primarily focusing on sociodemographic status and risk of an individual's return to the emergency department. Hefflinger and Hinshaw ${ }^{[7]}$ have reported that stigma exists at professional and institutional levels and is the reason for low health care utilization among children and adolescents with mental disorders seeking mental health services. The present study extends our understanding by examining the consequences for youth on being labelled with a psychiatric disorder when seeking health services for physical disorders. We have identified an important system-based gap in our services that can be easily remediated through education alone. 
As a result of the Kirby report ${ }^{[13]}$, greater attention is now focused on the mental well-being of children with psychiatric disorders. Our local response to the crisis as identified by Kirby has been to provide more psychiatric beds in hospitals. While a greater abundance of psychiatric services is required, according to the results of the present study, increasing access to services through increasing the number of psychiatric beds will in fact harm children in the absence of an integrated system wherein their physical disorders may be appropriately identified and treated. More research is required to fully understand the current inequity in the treatment of physical disorders of children labelled with psychiatric problems. Traditionally, children's mental health services within hospital settings have developed within organizational silos in relation to other health services wherein mental health consultation is at best a minor component. As it stands to further develop segregated systems of care, to address the mental and physical needs of children will simply perpetuate the current state of affairs. Given the propensity for our institutions to perpetuate the status quo, a new direction in policy is immediately required to guide the change.

\section{Presentation at a meeting}

Organization IACAPAP 2012, Place Paris, France, Date: July 21-July 25, 2012.

\section{Conflict of interest}

Both authors have no conflicts of interest to disclose.

\section{Acknowledgement and funding}

There are no other acknowledgments. This study was partly funded by the Norlien Foundation and the O'Brien Centre.

\section{References}

[1] Jacobs P, Yim R, Ohinmaa A. Expenditures on mental health and addictions for Canadian provinces in 2003 and 2004 . Can J Psychiatry. 2008; 53(5): 306-13. PMid: 18551851.

[2] Pescosolido BA, Jensen PS, Martin JK, et al. Public knowledge and assessment of child mental health problems: findings from the national stigma study-children. J Am Acad Child Adolesc Psychiatry. 2008; 47(3): 339-49. PMid: 18216729.

http://dx.doi.org/10.1097/CHI.0b013e318160e3a0

[3] Burns BJ, Costello EJ, Angold A, et al. Children's mental health service use across service sectors. Health Aff. (Millwood). 1995; 14(3): 147-159. http://dx.doi.org/10.1377/hlthaff.14.3.147

[4] Link BG, Cullen FT, Struening E, et al. A modified labeling theory approach to mental disorders: an empirical assessment. Am Sociol Rev. 1989; 54(3): 400-423. http://dx.doi.org/10.2307/2095613

[5] Kazdin AE, Holland L, Crowley M. Family experience of barriers to treatment and premature termination from child therapy. J Consul Clin Psychol. 1997; 65: 453-463. http://dx.doi.org/10.1037/0022-006X.65.3.453

[6] Unites States department of health and human services. Mental health: a report of the surgeon general. Rockville, MD: Department of Health and Human Services, National Institute of Mental Health, 1999.

[7] Heflinger CA, Hinshaw SP. Stigma in child and adolescent mental health services research: understanding professional and institutional stigmatization of youth with mental health problems and their families. Adm Policy Ment Health. 2010; 37(1-2): 61-70. PMid: 20232133. http://dx.doi.org/10.1007/s10488-010-0294-z

[8] Cawthorpe D. (submitted) Population, policy and stigma: a novel health index. J Epidemiol Community Health. 2012.

[9] Wilkes C, Guyn L, Li B, et al. Association of child and adolescent psychiatric disorders with somatic or biomedical diagnoses: do population-based utilization study results support the adverse childhood experiences study? Perm J. 2012; 16(2): 23-26. PMid: 22745612. http://dx.doi.org/10.7812/TPP/11-149

[10] Newton AS, Rosychuk RJ, Dong K, et al. Emergency health care use and follow-up among sociodemographic groups of children who visit emergency departments for mental health crises. CMAJ. 2012; 184(12): E665-E674. PMid: 22690003. http://dx.doi.org/10.1503/cmaj.111697

[11] Kutcher S, Davidson S. Mentally ill youth: meeting service needs. CMAJ. 2007; 176(4): 417. PMid: 17296945. http://dx.doi.org/10.1503/cmaj.061694

[12] Wilkes C, Cawthorpe D. The need for more children's mental health services. CMAJ. 2008; 178(11): 1465-1466. PMid: 18490643. http://dx.doi.org/10.1503/cmaj.1080017

[13] Kirby MJL, Keon WJ. Out of the shadows at last: transforming mental health, mental illness and addiction services in Canada. Ottawa (ON): the standing senate committee on social affairs, science and technology; 2006. Available from: http://www.parl.gc.ca/Content/SEN/Committee/391/soci/rep/rep02may06-e.htm (accessed July 03, 2012). 